\title{
Eculizumab in the management of paroxysmal nocturnal hemoglobinuria: patient selection and special considerations
}

This article was published in the following Dove Press journal:

Therapeutics and Clinical Risk Management

I August 2016

Number of times this article has been viewed

\author{
Fatimah Al-Ani' \\ Ian Chin-Yee' \\ Alejandro Lazo-Langner ${ }^{1,2}$ \\ 'Department of Medicine, Division \\ of Hematology, ${ }^{2}$ Department of \\ Epidemiology and Biostatistics, \\ Western University, London, ON, \\ Canada
}

\begin{abstract}
Paroxysmal nocturnal hemoglobinuria $(\mathrm{PNH})$ is a nonmalignant clonal disorder resulting from somatic mutation in the $P I G-A$ gene leading to a deficiency of the membraneanchoring molecule glycosylphosphatidylinositol. The lack of expression of two glycosylphosphatidylinositol-anchored proteins involved in the regulation of the complement system renders PNH erythrocytes susceptible to complement-mediated lysis. Clinical manifestations include thromboembolic disease, chronic kidney injury, pulmonary hypertension, smooth muscle dysfunction, and chronic hemolysis. Until recently, treatment was mainly supportive with most patients suffering from significant morbidity and shortened survival compared to age-matched controls. The development of eculizumab, a humanized monoclonal antibody directed against the terminal complement protein $\mathrm{C} 5$, has resulted in dramatic improvements of survival and reduction in complications. In this paper, we review some special considerations pertaining to the use of eculizumab for PNH.
\end{abstract}

Keywords: somatic mutation, GPI, survival, hemolysis, MDS, anemia

\section{Overview of paroxysmal nocturnal hemoglobinuria}

Paroxysmal nocturnal hemoglobinuria $(\mathrm{PNH})$ is an acquired hemolytic anemia resulting from a somatic mutation in the $P I G-A$ gene in the hematopoietic stem cells. ${ }^{1}$ This mutation leads to a deficiency of glycosylphosphatidylinositol (GPI) membraneanchoring proteins on the surface of PNH cells. Two of these anchoring proteins, CD55 and CD59, are complement inhibitors, and the deficiency of such proteins will render PNH erythrocytes susceptible to complement-mediated lysis. ${ }^{2}$ Recently, new evidence on $\mathrm{PNH}$ pathogenesis supports the notion that the expansion of the $\mathrm{PNH}$ clone results from escape from T-cell-mediated autoimmune damage to hematopoietic stem cells, with the GPI molecule as a target. This might further consolidate the observed association between PNH and aplastic anemia (AA). ${ }^{3} \mathrm{PNH}$ is a very rare disease with a prevalence of $\sim 16$ per million people, ${ }^{4}$ and it can present at any age but mainly occurs in young adults, with the usual age at diagnosis being early $30 \mathrm{~s}^{4-6}$

Despite being rare, $\mathrm{PNH}$ is "the most vicious acquired thrombophilic state known", yet the mechanism of this virulent thrombotic behavior is still elusive. ${ }^{3,7}$ Thromboembolic (TE) events occur in $\sim 50 \%$ of PNH patients, and it is the main cause of death in approximately one-third of patients with $\mathrm{PNH}^{8}{ }^{8}$ The intravascular hemolysis in $\mathrm{PNH}$ is a critical factor in triggering thrombosis. The release of hemoglobin $(\mathrm{Hb})$ into the blood stream results in massive nitric oxide scavenging leading to vasospasm and to increase in platelets' reactivity and aggregation. ${ }^{9,10}$ Therefore, the process of nitric 
oxide depletion by the free $\mathrm{Hb}$ will not only predispose patients to developing thromboembolism but also lead to vascular constriction (manifested as pulmonary hypertension, renal dysfunction, and erectile dysfunction) and smooth muscle dystonia of the gastrointestinal tract (manifested as abdominal pain, dysphagia, and esophageal spasm). ${ }^{2,9,11}$ Additionally, renal dysfunction is also caused by chronic hemolysis-induced hemosiderin deposition in the proximal renal tubules, in addition to alterations in renal blood flow caused by nitric oxide depletion. ${ }^{9,10}$

Until a decade ago, the treatment options for $\mathrm{PNH}$ were mainly supportive including blood transfusion, erythropoiesis-stimulating agents, corticosteroids, anabolic steroids, iron therapy, thrombosis prophylaxis, and sometimes thrombolytic therapy or allogenic bone marrow transplantation. ${ }^{2,3}$ Eculizumab was first introduced in 2004 in a preliminary pilot study of eleven participants. ${ }^{12}$ It was found to dramatically reduce intravascular hemolysis and blood transfusion requirements. Despite the open-label design, the potential risk of bias was minimal as all the reported outcomes were objectively measurable. However, other issues including the small sample size, short-term follow-up, and the potential risk of selection bias (only transfusion-dependent patients were included) should be considered when interpreting the results of this study. Subsequently, two Phase III trials - the TRIUMPH and SHEPHERD studies - proved that eculizumab therapy for PNH is effective, safe, and well tolerated in a standardized setting with predefined outcome criteria. ${ }^{11,13}$ A total of 87 and 97 patients were enrolled in these studies, respectively, overcoming the small sample size issue of the pilot study. However, issues such as selection of only transfusion-dependent patients and failure to report on TE rates as most patients were receiving concomitant anticoagulants remained. Eculizumab was finally approved for the treatment of patients with PNH in 2007 by the US Food and Drug Administration and by the European Medicines Agency. ${ }^{2,8}$

In this review, we focus on the up-to-date established positive impacts of eculizumab on $\mathrm{PNH}$ and its potential adverse events, highlighting certain issues that need to be addressed with eculizumab use.

\section{Eculizumab}

Eculizumab is a humanized monoclonal antibody directed against the terminal complement protein $\mathrm{C} 5$, which blocks the formation of the membrane attack complex (C5-9), thus protecting PNH red blood cells from complementmediated intravascular hemolysis. ${ }^{3,14}$ Its effectiveness has been evidenced by reduction or elimination of the need for blood transfusion, decrease in the incidence of thrombosis, improvement in anemia and quality of life (QoL), and prolongation of overall survival (OS). ${ }^{1,8,11}$ Furthermore, due to its use, stem cell transplantation is only considered for cases of severe marrow failure. , $^{3,15}$

\section{Pharmacodynamics and pharmacokinetics}

An inhibition of C5 sufficient to prevent hemolysis occurs at eculizumab plasma concentrations in excess of $35 \mathrm{~g} / \mathrm{mL} .^{2}$ At therapeutic doses, maximum plasma concentrations of eculizumab are achieved within 1 hour of infusion. ${ }^{2}$ Eculizumab reduces hemolysis in $\sim 1$ week, and its half-life elimination is $\sim 11$ days (range: $8-15$ days). The clearance for a typical PNH patient weighing $70 \mathrm{~kg}$ is $\sim 22 \mathrm{~mL} / \mathrm{h}$, and the volume of distribution is $\sim 7.7$ L. ${ }^{16}$

\section{Dose and schedule}

For PNH, eculizumab is administered as a weekly dose of $600 \mathrm{mg}$ by intravenous infusion for 35 minutes from week 1 to week 4, followed by infusion of $900 \mathrm{mg}$ at week 5 , and then $900 \mathrm{mg}$ every 14 days for an indefinite period. ${ }^{1,2}$

\section{Eculizumab and PNH - clinical efficacy}

The effect of eculizumab on PNH patients has dramatically changed the clinicians' management and patients' perspective toward this disease.

\section{Reducing blood transfusion requirement and stabilizing $\mathrm{Hb}$ level}

Symptoms of PNH are often disproportionate to the degree of anemia. ${ }^{11}$ However, achieving sustained stability of $\mathrm{Hb}$ and transfusion independence were the main outcomes measured with eculizumab therapy in PNH studies. ${ }^{11-13}$ Those two outcomes were chosen because both are measurable, and can be easily compared with pre- or no-eculizumab treatment end points to assess its response. Studies assessing the transfusion requirement and transfusion independence rates with eculizumab are illustrated in Table 1.

In the TRIUMPH study, 43 patients on eculizumab were compared with 44 patients on placebo in a double-blind randomized controlled trial, with the outcomes being the stabilization of $\mathrm{Hb}$ and transfusion independence. ${ }^{11} \mathrm{Hb}$ stabilization occurred in $49 \%$ in the eculizumab group compared to $0 \%$ in the placebo group $(P<0.001)$. Furthermore, there was a significant reduction in the median number of units transfused in eculizumab group as compared to the placebo group. Similar findings were also reported in the SHEPHERD study. ${ }^{13}$

Kelly et al evaluated 79 consecutive $\mathrm{PNH}$ patients over 8 years, and compared them with 30 matched historical 
Table I Studies assessing hematological response and transfusion requirement in $\mathrm{PNH}$ patients treated with eculizumab

\begin{tabular}{|c|c|c|c|c|c|c|c|}
\hline Study & Total $\mathbf{N}$ & Hb stabilization & $\begin{array}{l}\text { Median number of } \\
\text { PRBC units transfused } \\
\text { (eculizumab vs placebo) }\end{array}$ & $P$-value & $\begin{array}{l}\text { Overall } \\
\text { transfusion } \\
\text { reduction }\end{array}$ & $\begin{array}{l}\% \text { of transfusion } \\
\text { independence }\end{array}$ & $\begin{array}{l}\text { Transfusion } \\
\text { reduction in } \\
\text { dependent group }\end{array}$ \\
\hline TRIUMPH"I & 87 & $49 \%(P<0.00 \mathrm{I})$ & 0 vs 10 & $<0.001$ & $73 \%$ & $51 \%$ (at 6 months) & Yes \\
\hline SHEPHERD ${ }^{13}$ & 96 & - & 0 vs 8 & $<0.001$ & $52 \%$ & $51 \%$ (at I 2 months) & - \\
\hline Kelly et $\mathrm{al}^{8}$ & 79 & - & 5 vs 19 (mean number) & $<0.001$ & - & $66 \%$ (at 6 months) & Yes $(P<0.03)$ \\
\hline Hillmen et al ${ }^{17}$ & 195 & - & - & - & $54.7 \%$ & $\begin{array}{l}82 \% \text { (at the last } 6 \text { months } \\
\text { of study) }\end{array}$ & Yes $(P<0.00 \mathrm{I})$ \\
\hline AEGIS ${ }^{18}$ & 29 & $\begin{array}{l}31 \% \mathrm{Hb} \text { increase } \\
(P<0.00 \mathrm{I})\end{array}$ & 0.7 vs 4.9 & 0.0016 & - & $96 \%$ (at 18-24 months) & - \\
\hline
\end{tabular}

Abbreviations: $\mathrm{PNH}$, paroxysmal nocturnal hemoglobinuria; Hb, hemoglobin; PRBC, packed red blood cell.

controls from the pre-eculizumab era. ${ }^{8}$ The 12 -month mean transfusion requirement was significantly reduced in patients receiving eculizumab compared to controls. Although this study was non-randomized, the inclusion of consecutive patients might reduce the risk of bias.

Long-term safety and efficacy data of eculizumab have been reported in two studies: the study by Hillmen et al and AEGIS study. ${ }^{17,18}$ The first study included a total of 195 patients from three Phase III trials with a maximum duration of 66 months, and reported a total transfusion independence in $82 \%$ of patients. ${ }^{17}$ The AEGIS trial was an open-label, noncomparative, multicenter Phase II study of 29 Japanese PNH patients, followed by a further 2-year extension study. ${ }^{18}$ Only 27 patients were included in the extension study with a total transfusion independence of $96 \%{ }^{17,18}$ The importance of the AEGIS extension study lies in the fact that it is the first study reporting long-term data of eculizumab in a trial setting among Asian PNH patients, a population in which PNH seems to be more frequent than in the Western population. ${ }^{19}$

In patients in whom transfusion independence was not reached, the TRIUMPH trial ${ }^{11}$ and the studies by Kelly et al ${ }^{8}$ and Hillmen et al ${ }^{17}$ reported similar findings of reduction in the number of red cell units transfused after starting eculizumab as compared to before eculizumab treatment.

Finally, a recent retrospective study included $123 \mathrm{PNH}$ patients treated with eculizumab and 191 historical controls from the French registry. Although the transfusion requirements were not reported in the study, the authors did suggest that anemia could persist in some patients. ${ }^{1}$

\section{Improving QoL}

QoL is affected in the majority of patients with PNH with $96 \%$ complaining of fatigue and $>40 \%$ having symptoms of smooth muscle spasm often of sufficient severity to require narcotic analgesics. ${ }^{20}$ Fatigue is multifactorial and is not simply the result of anemia, as $60 \%$ of these patients are transfusion independent. ${ }^{20}$ The fluctuation in $\mathrm{Hb}$ and the inconvenience of the frequent blood transfusions may also contribute to the impaired QoL. Nevertheless, eculizumab has impacted favorably on the QoL in both transfusion-dependent and transfusion-independent patients. ${ }^{3,9,11,21,22}$

In the TRIUMPH study, QoL assessments were performed with the use of two instruments: the Functional Assessment of Chronic Illness Therapy - Fatigue (FACIT-F) scale and the European Organization for Research and Treatment of Cancer Quality of Life Questionnaire. Patients in the eculizumab group had a mean increase (improvement) in scores on the FACIT-F of $6.4 \pm 1.2$ points from baseline to week 26 , compared to the placebo group in which the mean score decreased by $4.0 \pm 1.7$ points during this period $(P<0.001) .{ }^{11}$ Importantly, only transfusion-dependent patients were included, and QoL was assessed prior to transfusion and after eculizumab, without posttransfusion assessments. Therefore, it is not possible to fully determine whether differences are a result of eculizumab or the anemia correction. ${ }^{2}$ However, improvement in the FACIT-F score was also noted independent of improvement in $\mathrm{Hb}$ level in patients receiving eculizumab as compared to placebo, supporting the positive impact of eculizumab on QoL regardless of $\mathrm{Hb}$ level.

With respect to the European Organization for Research and Treatment of Cancer Quality of Life Questionnaire, the eculizumab group had overall significant improvements in scores of functional status, fatigue, pain, dyspnea, loss of appetite, and insomnia, compared to placebo. ${ }^{11}$ Similarly, improvements were demonstrated in several QoL parameters including physical, emotional, and cognitive functioning, global health, fatigue, dyspnea, and insomnia. ${ }^{12}$ Similarly, the AEGIS study also showed a consistent improvement in QoL in the majority of patients with sustained reductions in fatigue and dyspnea. ${ }^{18}$

Of note, $\sim 25 \%$ of PNH patients treated with eculizumab still need recurrent blood transfusions; however, even these patients report improved QoL. ${ }^{3}$ Improvements in symptoms including abdominal pain, dysphagia, and erectile 
dysfunction have been reported during eculizumab therapy despite the observation that total $\mathrm{Hb}$ levels were unchanged or not completely corrected. ${ }^{11,12,21}$ This supports the notion that intravascular hemolysis plays an important role in diminishing QoL in PNH patients. ${ }^{11}$

\section{Reducing intravascular hemolysis}

As previously mentioned, intravascular hemolysis results in symptoms of smooth muscle dystonia, including abdominal pain, dysphagia, and erectile dysfunction. ${ }^{3,11}$ Eculizumab therapy results in a reduction of hemolysis and a new steadystate $\mathrm{Hb}$ level, due to balance of the underlying bone marrow dysfunction, the increased half-life of PNH erythrocytes, and the new level of transfusions (if any) required. ${ }^{11}$ High percentages of PNH-type red blood cells have been observed after eculizumab treatment, indicating that these cells have not undergone hemolytic destruction. ${ }^{10,18}$

Lactate dehydrogenase (LDH), a biochemical marker of hemolysis, is used frequently to assess intravascular hemolytic activity. In the pre-eculizumab era, $\mathrm{PNH}$ patients with hemolysis ( $\mathrm{LDH} \geq 1.5 \times$ upper limit of normal) had a 4.8 -fold increase in mortality compared to the general population. ${ }^{23}$ The TRIUMPH study showed a rapid, significant, and sustained reduction in the LDH to normal or near-normal levels in patients on eculizumab. ${ }^{11}$ Other studies have reported similar findings that last beyond 8 years of eculizumab therapy, reflecting inhibition of chronic hemolysis. ${ }^{8,17,18}$

\section{Preventing thrombosis}

Thromboembolism occurs in 50\% of patients, and represents the major cause of death in $\mathrm{PNH} .{ }^{6,24,25}$ Once an initial thrombosis occurs, the risk of further thrombotic events increases, with a five- to ten-fold increase in mortality. ${ }^{8}$ Interestingly, TE events in $\mathrm{PNH}$ are not restricted to the venous system. ${ }^{4,6,22}$ Eculizumab was able to show a substantial impact in lowering the risk of thrombosis in $\mathrm{PNH}$ as summarized in Table 2 .

It has been shown that patients on eculizumab have a significant and sustained decrease in activation of both the plasmatic hemostatic system and the endothelium. ${ }^{26} \mathrm{Com}-$ pared to pretreatment values, patients on eculizumab were found to have lower levels of coagulation factors such as von Willebrand factor, of by-products of coagulation such as D-dimer, and also of endothelial cell molecules such as vascular cell adhesion molecule $1 .^{26}$ These changes might be due to the blocking effect of eculizumab on $\mathrm{C} 5$, thus reducing platelet and endothelial activation, intravascular hemolysis, and release of thromboplastin-like substances. ${ }^{7,27}$
Table 2 Studies assessing thromboembolism rates with eculizumab

\begin{tabular}{|c|c|c|c|}
\hline Study & $\begin{array}{l}\text { TE event rate/ } 100 \text { PYs } \\
\text { with eculizumab vs } \\
\text { without eculizumab }\end{array}$ & $P$-value & Notes \\
\hline Hillmen et $\mathrm{al}^{22}$ & 0.72 vs II.54 & $<0.001$ & \\
\hline Kelly et $\mathrm{al}^{8}$ & 0.8 vs 6.5 & $<0.001$ & \\
\hline AEGIS 18 & Zero TE events & & \\
\hline Hillmen et $\mathrm{al}^{17}$ & $\begin{array}{l}3.6 \% \text { vs } 32.6 \%^{a} \\
2.3 \text { vs } 7.3^{b}\end{array}$ & & $\begin{array}{l}96.4 \% \text { of total } \\
\text { patients free of } \\
\text { thromboembolism }\end{array}$ \\
\hline Loschi et al' & $4 \%$ vs $29 \%^{a}$ & & \\
\hline
\end{tabular}

Notes: aPercentage, not rate. ${ }^{\text {bncidence rate calculated from information available }}$ in the study.

Abbreviations: TE, thromboembolic; PYs, person-years.

Initial studies were not designed to establish the effect of eculizumab on thrombosis as most trial patients were receiving concomitant anticoagulants. ${ }^{2,12,13}$ However, in later studies, eculizumab has consistently shown a protective effect against thrombosis. ${ }^{8,17,22,26}$ In a prespecified analysis, data from three combined studies ${ }^{11-13}$ showed that TE rate in patients receiving exclusively anticoagulants was reduced from 11.54 events per 100 patient-years before eculizumab treatment to 0.72 events per 100 patient-years during eculizumab treatment $(P<0.001)$, with a relative risk reduction of $94 \% .{ }^{22}$ Despite heterogeneity in the individual pre-study TE event rates, eculizumab treatment consistently resulted in a marked reduction in thromboembolism (Table 2). Other studies have also reported dramatic reductions in thrombosis after initiating eculizumab therapy ( 0.8 vs 5.6 events per 100 patient-years; $P<0.001)$ even after accounting for the shorter duration of follow-up since starting eculizumab. ${ }^{8}$ Of note, in the latter study, $50 \%$ of the pre-eculizumab thrombosis events occurred on anticoagulants, which supports the notion that in PNH patients with a previous history of thrombosis, anticoagulation alone may not be sufficient in preventing thromboses. On the other hand, after eculizumab treatment, primary prophylaxis with warfarin was stopped, whereas anticoagulation for secondary prophylaxis was not routinely stopped. Thus, cessation of anticoagulation in patients on eculizumab with no prior history of thrombosis is the current recommended practice in some centers. ${ }^{8}$ Similar TE reductions have been reported in other studies. ${ }^{17,18}$ TE events decreased by $81.8 \%$, with $96.4 \%$ of patients remaining free of TE events. ${ }^{17}$ In the AEGIS study, no TE events were reported during the 2-year extension period. ${ }^{18}$

A recent study from the French registry also provided evidence for significantly lower TE rates in patients on eculizumab compared to historical controls ( $4 \%$ vs $29 \%$ ). 
However, the study showed that thromboembolism remains a major issue in PNH, even in the eculizumab era, as highlighted by the negative impact of a previous thromboembolism on OS in adjusted analysis (hazard ratio [HR] 4.48, 95\% confidence interval $[\mathrm{CI}] 2.46-8.16 ; P<0.001) .{ }^{1}$

\section{Improving renal function}

Renal dysfunction has been well reported in $\mathrm{PNH},{ }^{11,18}$ and patients with $\mathrm{PNH}$ have a sixfold greater risk of chronic kidney disease (CKD) compared to the general population. ${ }^{9,11,18}$ More importantly, CKD is associated with an elevated risk of premature mortality contributing to $8 \%-18 \%$ of PNH-related deaths. ${ }^{6,17}$ Renal impairment in $\mathrm{PNH}$ is multifactorial and involves a direct effect of nitric oxide depletion, induced by cell-free $\mathrm{Hb}$, on the glomerular filtration rate and on renal plasma flow, and the repetitive exposure to cell-free $\mathrm{Hb}$ leading to chronic hemosiderin deposition in the proximal renal tubules. ${ }^{9,10}$ Supportive care measures do not prevent the occurrence or the progression of CKD. However, by inhibiting terminal complement activation and intravascular hemolysis, treatment with eculizumab significantly improves renal function. Several studies have shown consistently that eculizumab has a protective role leading to improvement in, or less progression of, renal disease. ${ }^{9,10,17}$

Hillmen et al provided evidence that sustained longterm therapy with eculizumab can reverse early-stage renal damage sustained prior to treatment initiation, particularly when administered early and prior to advanced renal impairment. ${ }^{17}$ In this study, CKD stages ${ }^{28}$ were assessed following each 6-month eculizumab treatment period. Continued time-dependent improvement in CKD was observed with sustained eculizumab treatment, with $24.4 \%$ of patients showing improvement in CKD after only 6 months of treatment and $45 \%$ of patients showing improvement after 36 months. Overall, patients were significantly more likely to experience an improvement than a worsening in renal function $(P=0.015) .{ }^{17}$

Similar findings have been reported in the AEGIS study. Sustained administration of eculizumab in patients with CKD at baseline was associated with improved renal function in $71 \%$, whereas $29 \%$ had their renal function stabilized. Eculizumab also improved renal function, particularly if administered early in the course of disease and before the kidney is more severely impaired. Improvements in renal function were observed in $88 \%$ of patients with stage $1 \mathrm{CKD}$, $67 \%$ of patients with stage $2 \mathrm{CKD}$, and $33 \%$ of patients with stages $3-4$ CKD. ${ }^{18}$
A large cohort of 195 PNH patients from parent eculizumab clinical trials was also assessed for their kidney function by Hillmen et al. ${ }^{9}$ In this study, improvement in renal function was more commonly seen in patients with baseline CKD stages 1-2 (67.1\% improvement), although improvement was also observed in patients with CKD stages 3-4. Overall, 40 (21\%) of 195 patients who demonstrated renal dysfunction or damage at baseline were no longer classified as such after 18 months of treatment. ${ }^{9}$

\section{Improving OS}

Historical studies have shown the median survival in $\mathrm{PNH}$ patients to be between 10 and 15 years from the time of diagnosis. ${ }^{4}$ More recently, data from a French series of patients in the pre-eculizumab era showed an improvement in median survival to 22 years, which is probably the result of improvements in supportive care and in the management of disease complications. ${ }^{25}$ With the use of eculizumab, the OS of PNH patients has consistently shown a remarkable improvement, approaching that of the general population. ${ }^{1,8,17}$ The survival rate of PNH patients in eculizumab studies was $97.6 \%$ at 3 years, ${ }^{17} 95.5 \%$ at 5 years $(P<0.03$ as compared to eculizumab-untreated patients), ${ }^{8}$ and $92 \%$ at 6 years $\left(P=0.037\right.$ as compared with controls). ${ }^{1,8,17}$ Furthermore, Kelly et al reported no significant difference in OS between the PNH patients treated with eculizumab compared with sex- and age-matched controls from the UK population. ${ }^{8}$ Another study suggested that the two factors statistically associated with a worse OS were the absence of treatment with eculizumab (HR 6.26, 95\% CI 2.72-14.43; $P<0.001$ ) and a previous history of TE events (HR 4.48, 95\% CI 2.46-8.16; $P<0.001){ }^{1}$

\section{Absent or inadequate response to eculizumab in $\mathrm{PNH}$}

It has been reported that, with eculizumab, up to two-thirds of PNH patients who are transfusion dependent become transfusion independent. ${ }^{3}$ However, $\sim 25 \%$ of them still need recurrent albeit less frequent blood transfusion. ${ }^{3}$ The authors suggested that these heterogeneous hematological responses may be related to factors including underlying $\mathrm{AA},{ }^{1-3} \mathrm{C} 3$-mediated extravascular hemolysis, $, 3,15,29$ breakthrough hemolysis, ${ }^{30}$ or the presence of a specific mutation in the $\mathrm{C} 5$ gene which prevents eculizumab from binding to $\mathrm{C} 5$ protein. ${ }^{6}$

\section{The association of $\mathrm{PNH}$ with $\mathrm{AA}$}

In some patients, a poor or suboptimal response to eculizumab may indicate that the extent to which bone marrow 
failure was contributing to anemia had been underestimated. ${ }^{3}$ There is a close relationship between AA and $\mathrm{PNH}$, and a history of severe AA is present in at least one-half of all newly diagnosed PNH cases, ${ }^{31}$ whereas $\mathrm{PNH}$ clones are found in up to $70 \%$ of patients with AA. ${ }^{2,31}$ Recently, new evidence on $\mathrm{PNH}$ pathogenesis supports the notion that expansion of the PNH clone results from escape from T-cell-mediated autoimmune damage to normal hematopoietic stem cells, with the GPI molecule as target, consolidating further the observed association between PNH and AA. Indeed, GPIspecific $\mathrm{CD}^{+} \mathrm{T}$ cells - which have been identified in $\mathrm{PNH}$ patients - would spare selectively GPI-negative stem cells, thus enabling them to repopulate the marrow of a patient who would otherwise have AA. ${ }^{3}$

In the AEGIS trial, the effect of eculizumab was assessed in patients with $\mathrm{PNH}$ plus AA/myelodysplastic syndrome (MDS) and those with PNH alone, and no significant differences were found between the two groups in the end points assessed, namely hemolysis and blood transfusion. This finding suggests that patients with PNH and AA/MDS experience similar hemolysis-associated complications and can benefit from treatment with eculizumab. ${ }^{18}$

Another interesting observation has been reported by a case report of a PNH patient who was initially diagnosed with AA and treated subsequently with eculizumab. ${ }^{32}$ Erythropoiesis improved, and the patient became transfusion independent shortly after initiation of iron chelation with deferasirox. ${ }^{32}$ Similarly, the same finding has also been seen in MDS patients after starting deferasirox..$^{33,34}$ The significance of this management modality needs to be addressed in future studies.

It is too early to draw robust conclusions on the effect of eculizumab on patients with PNH plus AA, yet the evidence of the association between the two suggests that an inadequate marrow response to anemia may be a contributing factor to the observed persistent anemia in $\mathrm{PNH}$ patients receiving eculizumab. More importantly as many as $25 \%$ of patients with a previous diagnosis of AA treated with immunosuppression may progress on to develop hemolytic PNH requiring therapy. ${ }^{35}$

\section{Extravascular hemolysis with eculizumab}

Two studies have described the development of "iatrogenic" extravascular hemolysis with eculizumab. ${ }^{15,29}$ Risitano et al have suggested that binding of C3 by PNH red blood cells may constitute an additional disease mechanism, strongly enhanced by eculizumab treatment and producing a variable degree of extravascular hemolysis. ${ }^{15}$ Eculizumab affects only the distal complement pathway, thus neutralizing the deficiency of CD59 on PNH red blood cells by inhibition of $\mathrm{C} 5$ cleavage, but since these are also deficient in CD55, upstream complement activation may lead to C3 opsonization leading to a gradual accumulation of $\mathrm{C} 3$ fragments on their surface. These C3-opsonized red blood cells will be susceptible to phagocytosis by macrophages in the reticuloendothelial system, resulting in extravascular hemolysis. ${ }^{3,7}$ This phenomenon may explain the residual low-level hemolysis occurring in some eculizumab-treated patients, ${ }^{29}$ indicated by a positive Coombs test (classically negative in untreated PNH). ${ }^{3,7,29}$

More importantly, researchers have suggested that this process may play a role in limiting anemia improvement because it develops at the same time that intravascular hemolysis ceases. ${ }^{3,7}$ However, fortunately in many cases, it seems to have little or no clinical relevance. ${ }^{7}$ This conclusion has also been supported by studies in which a dramatic reduction in transfusion requirements was observed, indicating that the phenomenon of extravascular hemolysis is of limited clinical importance for most patients. ${ }^{8,17}$ In the next decade, researchers may quest for a way to control extravascular hemolysis. ${ }^{7}$ It has been recommended that selective splenic artery embolization can be used to treat severe extravascular hemolysis, as an alternative to splenectomy. ${ }^{3}$ Of note, selective splenic artery embolization has also been used to reduce spleen size and alleviate cytopenias in splenomegaly with hypersplenism in $\mathrm{PNH}$ patients. ${ }^{3}$

\section{Breakthrough hemolysis}

According to Nakayama et al, eculizumab-dosing intervals $>17$ days may be associated with an increased risk of developing breakthrough hemolysis in patients with $\mathrm{PNH}$ (odds ratio 1.6, 95\% CI 1.3-2.0; $P<0.01$ ). ${ }^{30}$ The recommended schedule is thus to maintain eculizumab administration every 14 days, although this needs to be further confirmed. ${ }^{1,2}$ Other factors may also account for breakthrough hemolysis such as pregnancy (detailed in the "Eculizumab in pregnancy" section).

\section{C5 gene mutation}

C5 gene mutation has been detected among Japanese patients with $\mathrm{PNH}$, identified as having poor response to eculizumab. ${ }^{6}$ A poor response was defined as markedly high levels of $\mathrm{LDH}$ during treatment, indicating that intravascular hemolysis remained unaffected, regardless of the persistence or improvement in other laboratory findings or clinical symptoms. This mutation prevents binding and blockade 
by eculizumab while retaining the functional capacity of the mutant $\mathrm{C} 5$ to cause hemolysis. The prevalence of this mutation among the patients with PNH (3.2\%) was similar to that among healthy Japanese persons $(3.5 \%)$.

\section{Special considerations \\ Meningococcal meningitis and other infections}

Complement inhibition by eculizumab leads to impairment in neutrophil and monocyte function, consequently affecting their ability to clear infections by encapsulated organisms, such as Neisseria meningitidis. ${ }^{2,8}$ Patients who are to be treated with eculizumab must be vaccinated against $N$. meningitidis at least 2 weeks prior to starting treatment. ${ }^{2}$

The tetravalent meningococcal vaccine is available against subgroups A, C, W, and Y but not for serogroup B, which is the most common serotype isolated in meningococcal sepsis in areas such as the UK. ${ }^{8,9}$ This explains the reported rates of meningococcal infections in some studies despite all patients being vaccinated appropriately ${ }^{8,9}$ Hillmen et al and Kelly et al reported a rate of $1 \%$ and $2.5 \%$, respectively, for meningococcal meningitis on eculizumab. ${ }^{8,9}$ Therefore, in January 2010 , the UK policy changed to include antibiotic prophylaxis for all patients (penicillin V $500 \mathrm{mg}$ twice a day or erythromycin $500 \mathrm{mg}$ twice a day for patients intolerant to penicillin), in addition to the tetravalent meningococcal vaccination. ${ }^{8}$ In 2013, Bexsero ${ }^{\circledR}$ became the first broad-coverage vaccine to be licensed for active immunization against serotype B meningococcal disease.$^{36}$ Bexsero is now licensed in $>35$ countries worldwide for varying age groups, including the European Union, Australia, Brazil, Canada, Chile, Uruguay, and the USA, and it is usually combined with the tetravalent vaccine in $\mathrm{PNH}$ patients planned for eculizumab for full coverage of the five serotypes. ${ }^{36}$ However, in daily practice, clinicians may still prefer to continue on penicillin or amoxicillin prophylaxis while awaiting the vaccines to kick in, and some would still choose to continue penicillin even after vaccinated because other subtypes may not be covered.

Other infections have also been reported in eculizumab studies. The AEGIS study reported a total of 159 treatmentemergent infections, of which the majority were mild in severity (87.4\%). Only seven of the 159 infections (4\%) were considered probably related to eculizumab, of which three (one case each of pneumonia, cellulitis, and sepsis) were severe. There were no meningococcal infections reported. ${ }^{18}$ Hillmen et al also reported an overall infection rate of $12.8 \%{ }^{9}$

Overall, studies indicate that eculizumab is well tolerated over the long term, and its profile is consistently safe across studies. However, further precautions might be considered in immune-compromised categories such as elderly or neutropenic patients.

\section{Eculizumab in pregnancy}

The median age at diagnosis of PNH is 34 years, so affected females are often in the reproductive age group. ${ }^{37}$ Although $\mathrm{PNH}$ is not known to adversely affect female fertility, several fetal-maternal complications have been reported in pregnant PNH patients related to exacerbated intravascular hemolysis, including spontaneous miscarriages (45\%), premature births, increased blood transfusion requirements, thrombocytopenia, thromboembolism, and accelerated preeclampsia. ${ }^{37-39}$ In $\mathrm{PNH}$, maternal mortality has been reported to range between $8 \%$ and $21 \%$, mostly occurring in the postpartum period, and thromboembolism being the primary cause. On the other hand, fetal mortality has been reported to range between $4 \%$ and $9 \%$, mostly associated with premature births. ${ }^{38,40,41}$ Close monitoring of all pregnant patients with $\mathrm{PNH}$ is thus recommended.

Pregnancy activates the complement cascade, with increased terminal complex formation in the third trimester. ${ }^{14,42}$ Therefore, PNH has been considered a relative contraindication for pregnancy, and patients were usually discouraged from conceiving. ${ }^{37,39}$ Because of the risk of life-threatening thrombotic complications, pregnant women with $\mathrm{PNH}$ in the pre-eculizumab era were to receive full anticoagulation during pregnancy and for at least 4-6 weeks postpartum. ${ }^{38}$

Several studies and case reports have described the use of eculizumab in pregnant PNH patients..$^{32,37,39,43-45}$ It has been noted that the dose of eculizumab required to block complement during the later stages of pregnancy is greater than that required in the nonpregnant state. ${ }^{44}$ The increased activity of lysosomal enzyme, as well as the physiologic changes that occur during pregnancy, such as the physiologic expansion of intravascular volume, may account for the higher doses required..$^{32,39}$

In pregnant patients, the eculizumab schedule has been modified by reducing the interval between eculizumab dose administration to $\sim 7-12$ days, ${ }^{43-45}$ by administering higher doses of eculizumab (1,200 mg every 2 weeks during the third trimester), ${ }^{37}$ or by using both modalities together, ${ }^{32,39}$ according to physician discretion in order to control hemolysis as pregnancy progresses. ${ }^{32,37,39,43-45}$

The largest PNH study in pregnancy so far has been reported by Kelly et al in $2015 .{ }^{39}$ Data were reported on 75 pregnancies in 61 women with PNH from the International PNH Interest Group and the International PNH Registry. ${ }^{39}$ In 54\% of 
pregnancies that progressed past the first trimester, the dose or the frequency of use of eculizumab had to be increased. Requirements for transfusion of red blood cells increased during pregnancy, from a mean of 0.14 units per month in the 6 months before pregnancy to 0.92 units per month during pregnancy, and platelet transfusions were required in $21 \%$ of pregnancies, mostly administered at the time of delivery. ${ }^{39}$ Furthermore, premature birth rate of $29 \%$ was reported. Two TE events were documented, and both occurred during the postpartum period, despite that thromboprophylaxis with low-molecular weight heparin was used in the majority of patients $(88 \%)$. One of the thrombotic events occurred after a plasma infusion was administered to treat a postpartum hemorrhage. Plasma contains high levels of complement and can overcome the effects of eculizumab and thereby render the patient susceptible to complications of PNH. The use of plasma should probably be avoided if possible.

Initially, it was thought that eculizumab would cross the placenta and appear in breast milk. ${ }^{2}$ However, subsequent studies reported that eculizumab does not appear to cross the placenta nor it is excreted in breast milk. ${ }^{37,43-45}$ However, in a study on 21 cord-blood samples, eculizumab was detected in seven of the samples, whereas the drug was not detected in any of the ten breast milk samples tested. ${ }^{39}$ Recently, two studies suggested that if eculizumab crosses the placenta, the level is not high enough to affect complement activation. ${ }^{39,46}$

Overall, studies highlighted the significance of regular and frequent monitoring of hemolytic parameters in $\mathrm{PNH}$ patients during pregnancy to detect early disease activity. In the end, there are no adequate and well-controlled studies of eculizumab in pregnant women, and a firm conclusion is difficult to be drawn. Nevertheless, all studies available suggested that eculizumab provided benefit for women with PNH during pregnancy, as evidenced by a high rate of fetal survival, a low rate of fetomaternal complications, and a reduced rate of blood transfusion, compared to pregnant $\mathrm{PNH}$ patients not treated with eculizumab. ${ }^{37,39,43-45}$ It also appears that eculizumab can be safely used throughout pregnancy.

In summary, the recommended practice in pregnant women with $\mathrm{PNH}$ receiving eculizumab includes dose escalation and/or reduction in dose interval to control hemolysis, avoidance of the use of plasma if possible, and thromboprophylaxis with low-molecular weight heparin throughout the pregnancy up to 6 weeks following delivery, even with the use of eculizumab. However, whether combining thromboprophylaxis with eculizumab (specifically during the postpartum period) would be sufficient for preventing thromboembolism, or whether dose escalation is needed in high-risk patients, such as those having a prior history of thromboembolism or requiring $\mathrm{C}$-section, still remain as questions.

\section{Eculizumab in elderly}

The median age at diagnosis of PNH is in the early 30s, and thus, patients aged $>80$ years were rarely enrolled, yet $\mathrm{PNH}$ can still present at any age. ${ }^{5,6,37}$ In view of the known ageassociated reduction in immune function and the effect of eculizumab on the suppression of the terminal complement pathway, one concern is that treatment with eculizumab may elevate the risk of infection in elderly patients. ${ }^{10}$ In the TRIUMPH and SHEPHERD trials, there were no apparent age-related differences observed in patients aged 65 years or older treated with eculizumab. However, the number of those patients was only 15 , and clearly, this number would not be sufficient to determine whether they respond differently than younger patients. ${ }^{2}$

A case report by Ooe and Nagai in Japan has described the use of eculizumab in an 86-year-old PNH patient. ${ }^{10}$ Despite that poor prognosis was considered in this patient due to $\mathrm{PNH}$-induced chronic renal failure and concomitant underlying myelodysplasia, eculizumab use was associated with rapid suppression of hemolysis, and significant improvement in both blood transfusion requirement and renal function from CKD stage 4 to stage 3 with no infections reported.

Although findings from available studies suggest that eculizumab can be used safely in elderly PNH patients with proved efficacy, the overall experience of eculizumab in geriatric population is still very limited, and decisions should be made on a case-by-case basis.

\section{Risk of MDS/acute leukemia with eculizumab}

An important concern with eculizumab is the possibility of clonal evolutions arising from a prolonged complement blockade. One study reported no significant difference regarding clonal evolution to MDS or acute myeloid leukemia in both eculizumab and control cohorts. ${ }^{1}$ However, no conclusions can be drawn, since a longer follow-up will be necessary as the median time to clonal evolution is 10 years. ${ }^{1}$ Furthermore, the rate of clonal myeloid evolution into MDS or acute leukemia was reported to be $\sim 4 \%$ in $\mathrm{PNH}$ patients receiving eculizumab in another study, ${ }^{8}$ which is a similar rate reported in $\mathrm{PNH}$ patients before eculizumab therapy. ${ }^{6,25,26}$ Interestingly, in the study by Kelly et al, MDS and/or acute leukemia occurred within the GPI-negative clone of cells, indicating that the majority of hematopoiesis in these patients 
was derived from PNH stem cells. ${ }^{8}$ Long-term administration of eculizumab to Japanese patients with PNH showed that the burden of PNH disease and baseline characteristics were similar in patients with and without a history of bone marrow dysfunction such as AA or MDS. ${ }^{18}$ No cases of clonal evolution were reported by Hillmen et al. ${ }^{17}$

In fact, the effect of eculizumab on the development of clonal diseases is still unclear, and long-term data are definitely required.

\section{Paucity of long-term "real-world" data}

In view of the rarity of $\mathrm{PNH}$, and the relatively recent introduction and approval of eculizumab, long-term data are scarce. Perhaps, the best standardized long-term safety and efficacy data of eculizumab were presented by Hillmen et al. ${ }^{17}$ They investigated 195 patients with $\mathrm{PNH}$ who had participated in one of three prospective parent trials: the Phase II pilot study and its extensions - TRIUMPH and SHEPHERD trials. ${ }^{11-13}$ The median eculizumab treatment duration was 30.3 months, with a maximum duration of 66 months. Overall, long-term treatment with eculizumab resulted in sustained improvement in patient outcomes by rapidly reducing hemolysis and improving patient survival due to significant reduction in the frequency of severe and lifethreatening morbidities, such as TE events and CKD with a favorable safety profile. However, "real-world" data with long-term follow-up are still lacking. The International PNH Registry (www.pnhregistry.com) is providing further opportunities to continue studying and understanding the long-term benefits of eculizumab in patients with $\mathrm{PNH}$ outside the clinical trial setting. ${ }^{17}$

\section{Conclusion}

The advent of eculizumab has resulted in a dramatic improvement of the natural history of $\mathrm{PNH}$ patients with improvement in virtually every aspect of these patients' lives. This is supported by the available data about which some limitations should be noted. First, the size of available studies is relatively small but dictated by the rarity of the disease. Second, most (if not all) research is funded by the pharmaceutical industry, which conveys an inherent risk of bias. Third, availability of randomized data is scarce, and many studies use historical controls, also risking the introduction of bias. Fourth, the treatment is very costly limiting access to it. In spite of these limitations, the data available so far are strong enough (and differences quite striking in some cases) to support the use of this therapy, although independent confirmatory data are sorely needed.
Fifth, notwithstanding the available evidence, many questions remain unanswered, in particular regarding long-term safety (eg, infections, clonal evolution), optimal management of special populations (eg, pregnant, elderly), role of eculizumab in PNH/AA, and need/utility of clinical and laboratory monitoring, among others. Finally, since the main deterrent to a wide adoption of this therapy is mainly of an economic nature and many patients who might benefit from it are unable to access this treatment, patient support programs and access to public funding as well as novel and more accessible agents are highly desirable.

\section{Disclosure}

ALL and ICY have received research funding from Alexion Pharmaceuticals in the past but none at the time of the preparation of this manuscript. ICY is a member of the Canadian PNH Network which is sponsored in part by Alexion Pharma Canada. No professional writing services were employed. Alexion Pharma (or its employees) was (were) not involved at any point in any aspect of the manuscript, from conception to submission. FAA reports no conflicts of interest in this work.

\section{References}

1. Loschi M, Porcher R, Barraco F, et al. Impact of eculizumab treatment on paroxysmal nocturnal hemoglobinuria: a treatment versus no-treatment study. Am J Hematol. 2016;91(4):366-370.

2. Dmytrijuk A, Robie-Suh K, Cohen MH, Rieves D, Weiss K, Pazdur R. FDA report: eculizumab (Soliris) for the treatment of patients with paroxysmal nocturnal hemoglobinuria. Oncologist. 2008;13(9):993-1000.

3. Luzzatto L. Recent advances in the pathogenesis and treatment of paroxysmal nocturnal hemoglobinuria. F1000Res. 2016;5.

4. Hillmen P, Lewis SM, Bessler M, Luzzatto L, Dacie JV. Natural history of paroxysmal nocturnal hemoglobinuria. N Engl J Med. 1995;333(19): 1253-1258.

5. Moyo VM, Mukhina GL, Garrett ES, Brodsky RA. Natural history of paroxysmal nocturnal haemoglobinuria using modern diagnostic assays. Br J Haematol. 2004;126(1):133-138.

6. Nishimura J, Kanakura Y, Ware RE, et al. Clinical course and flow cytometric analysis of paroxysmal nocturnal hemoglobinuria in the United States and Japan. Medicine (Baltimore). 2004;83(3):193-207.

7. Luzzatto L, Risitano AM, Notaro R. Paroxysmal nocturnal hemoglobinuria and eculizumab. Haematologica. 2010;95(4):523-526.

8. Kelly RJ, Hill A, Arnold LM, et al. Long-term treatment with eculizumab in paroxysmal nocturnal hemoglobinuria: sustained efficacy and improved survival. Blood. 2011;117(25):6786-6792.

9. Hillmen P, Elebute M, Kelly R, et al. Long-term effect of the complement inhibitor eculizumab on kidney function in patients with paroxysmal nocturnal hemoglobinuria. Am J Hematol. 2010;85(8):553-559.

10. Ooe Y, Nagai T. Successful use of eculizumab in an 86-year-old patient with paroxysmal nocturnal hemoglobinuria in Japan. Springerplus. 2014;3:10.

11. Hillmen P, Young NS, Schubert J, et al. The complement inhibitor eculizumab in paroxysmal nocturnal hemoglobinuria. $N$ Engl J Med. 2006;355(12):1233-1243.

12. Hillmen P, Hall C, Marsh JC, et al. Effect of eculizumab on hemolysis and transfusion requirements in patients with paroxysmal nocturnal hemoglobinuria. N Engl J Med. 2004;350(6):552-559. 
13. Brodsky RA, Young NS, Antonioli E, et al. Multicenter phase 3 study of the complement inhibitor eculizumab for the treatment of patients with paroxysmal nocturnal hemoglobinuria. Blood. 2008; 111(4):1840-1847.

14. Thomas TC, Rollins SA, Rother RP, et al. Inhibition of complement activity by humanized anti-C5 antibody and single-chain Fv. Mol Immunol. 1996;33(17-18):1389-1401.

15. Risitano AM, Notaro R, Marando L, et al. Complement fraction 3 binding on erythrocytes as additional mechanism of disease in paroxysmal nocturnal hemoglobinuria patients treated by eculizumab. Blood. 2009;113(17):4094-4100.

16. Soliris ${ }^{\circledR}$ (Eculizumab) concentrated solution for intravenous infusion [prescribing information]. New Haven CT, USA: Alexion Pharmaceuticals, Inc.; 2015. Available from: www.Soliris.net. Accessed May 23, 2016.

17. Hillmen P, Muus P, Roth A, et al. Long-term safety and efficacy of sustained eculizumab treatment in patients with paroxysmal nocturnal haemoglobinuria. Br J Haematol. 2013;162(1):62-73.

18. Kanakura Y, Ohyashiki K, Shichishima T, et al. Long-term efficacy and safety of eculizumab in Japanese patients with PNH: AEGIS trial. Int J Hematol. 2013;98(4):406-416.

19. Pramoonjago P, Pakdeesuwan K, Siripanyaphinyo U, Chinprasertsuk S, Kinoshita T, Wanachiwanawin W. Genotypic, immunophenotypic and clinical features of Thai patients with paroxysmal nocturnal haemoglobinuria. Br J Haematol. 1999; 105(2):497-504.

20. Meyers G, Weitz I, Lamy T, et al. Disease-related symptoms reported across a broad population of patients with paroxysmal nocturnal hemoglobinuria. Blood. 2007;110(11):3683 [abstract].

21. Hill A, Rother RP, Hillmen P. Improvement in the symptoms of smooth muscle dystonia during eculizumab therapy in paroxysmal nocturnal hemoglobinuria. Haematologica. 2005;90(12 Suppl):ECR40.

22. Hillmen $P$, Muus $P$, Duhrsen U, et al. Effect of the complement inhibitor eculizumab on thromboembolism in patients with paroxysmal nocturnal hemoglobinuria. Blood. 2007;110(12):4123-4128.

23. Jang JH, Kim JS, Yoon SS, et al. Predictive factors of mortality in population of patients with paroxysmal nocturnal hemoglobinuria (PNH): results from a Korean PNH registry. J Korean Med Sci. 2016; 31(2):214-221.

24. Araten DJ, Thaler HT, Luzzatto L. High incidence of thrombosis in African-American and Latin-American patients with Paroxysmal Nocturnal Haemoglobinuria. Thromb Haemost. 2005;93(1):88-91.

25. de Latour RP, Mary JY, Salanoubat C, et al. Paroxysmal nocturnal hemoglobinuria: natural history of disease subcategories. Blood. 2008;112(8):3099-3106.

26. Helley D, de Latour RP, Porcher R, et al. Evaluation of hemostasis and endothelial function in patients with paroxysmal nocturnal hemoglobinuria receiving eculizumab. Haematologica. 2010;95(4): 574-581.

27. Markiewski MM, Nilsson B, Ekdahl KN, Mollnes TE, Lambris JD. Complement and coagulation: strangers or partners in crime? Trends Immunol. 2007;28(4):184-192.

28. National Kidney Foundation. K/DOQI clinical practice guidelines for chronic kidney disease: evaluation, classification, and stratification. $\mathrm{Am}$ J Kidney Dis. 2002;39(2 Suppl 1):S1-S266.
29. Hill A, Rother RP, Arnold L, et al. Eculizumab prevents intravascular hemolysis in patients with paroxysmal nocturnal hemoglobinuria and unmasks low-level extravascular hemolysis occurring through $\mathrm{C} 3$ opsonization. Haematologica. 2010;95(4):567-573.

30. Nakayama H, Usuki K, Echizen H, Ogawa R, Orii T. Eculizumab dosing intervals longer than 17 days may be associated with greater risk of breakthrough hemolysis in patients with paroxysmal nocturnal hemoglobinuria. Biol Pharm Bull. 2016;39(2):285-288.

31. Schrezenmeier H, Muus P, Socie G, et al. Baseline characteristics and disease burden in patients in the International Paroxysmal Nocturnal Hemoglobinuria Registry. Haematologica. 2014;99(5):922-929.

32. Patriquin C, Leber B. Increased eculizumab requirements during pregnancy in a patient with paroxysmal nocturnal hemoglobinuria: case report and review of the literature. Clin Case Rep. 2015;3(2):88-91.

33. Jensen PD, Heickendorff L, Pedersen B, et al. The effect of iron chelation on haemopoiesis in MDS patients with transfusional iron overload. Br J Haematol. 1996;94(2):288-299.

34. Sanford D, Hsia CC. A case of transfusion independence in a patient with myelodysplastic syndrome using deferasirox, sustained for two years after stopping therapy. Curr Oncol. 2015;22(2):e128-e132.

35. Sugimori C, Chuhjo T, Feng X, et al. Minor population of CD55-. Blood. 2006;107(4):1308-1314.

36. Watson PS, Turner DP. Clinical experience with the meningococcal B vaccine, Bexsero( $(\mathbb{R})$ : prospects for reducing the burden of meningococcal serogroup B disease. Vaccine. 2016;34(7):875-880.

37. Sharma R, Keyzner A, Liu J, Bradley T, Allen SL. Successful pregnancy outcome in paroxysmal nocturnal hemoglobinuria $(\mathrm{PNH})$ following escalated eculizumab dosing to control breakthrough hemolysis. Leuk Res Rep. 2015;4(1):36-38.

38. Ray JG, Burows RF, Ginsberg JS, Burrows EA. Paroxysmal nocturnal hemoglobinuria and the risk of venous thrombosis: review and recommendations for management of the pregnant and nonpregnant patient. Haemostasis. 2000;30(3):103-117.

39. Kelly RJ, Hochsmann B, Szer J, et al. Eculizumab in pregnant patients with paroxysmal nocturnal hemoglobinuria. N Engl J Med. 2015; 373(11):1032-1039.

40. de Guibert S, Peffault de Latour R, Varoqueaux N, et al. Paroxysmal nocturnal hemoglobinuria and pregnancy before the eculizumab era: the French experience. Haematologica. 2011;96(9):1276-1283.

41. Bais J, Pel M, von dem Borne A, van der Lelie H. Pregnancy and paroxysmal nocturnal hemoglobinuria. Eur J Obstet Gynecol Reprod Biol. 1994;53(3):211-214.

42. Townsley DM, Young NS. Blood consult: paroxysmal nocturnal hemoglobinuria and its complications. Blood. 2013;122(16):2795-2798.

43. Danilov AV, Brodsky RA, Craigo S, Smith H, Miller KB. Managing a pregnant patient with paroxysmal nocturnal hemoglobinuria in the era of eculizumab. Leuk Res. 2010;34(5):566-571.

44. Kelly R, Arnold L, Richards S, et al. The management of pregnancy in paroxysmal nocturnal haemoglobinuria on long term eculizumab. Br J Haematol. 2010;149(3):446-450.

45. Marasca R, Coluccio V, Santachiara R, et al. Pregnancy in PNH: another eculizumab baby. Br J Haematol. 2010;150(6):707-708.

46. Hallstensen RF, Bergseth G, Foss S, et al. Eculizumab treatment during pregnancy does not affect the complement system activity of the newborn. Immunobiology. 2015;220(4):452-459.
Therapeutics and Clinical Risk Management

\section{Publish your work in this journal}

Therapeutics and Clinical Risk Management is an international, peerreviewed journal of clinical therapeutics and risk management, focusing on concise rapid reporting of clinical studies in all therapeutic areas, outcomes, safety, and programs for the effective, safe, and sustained use of medicines. This journal is indexed on PubMed Central, CAS,

\section{Dovepress}

EMBase, Scopus and the Elsevier Bibliographic databases. The manuscript management system is completely online and includes a very quick and fair peer-review system, which is all easy to use. Visit http://www.dovepress.com/testimonials.php to read real quotes from published authors. 